\title{
BMJ Open Is self-monitoring of blood glucose effective in improving glycaemic control in type 2 diabetes without insulin treatment: a meta-analysis of randomised controlled trials
}

\author{
Hongmei Zhu, ${ }^{1}$ Yanan Zhu, ${ }^{1}$ Siu-wai Leung ${ }^{1,2}$
}

To cite: Zhu H, Zhu Y, Leung S-w. Is selfmonitoring of blood glucose effective in improving glycaemic control in type 2 diabetes without insulin treatment: a meta-analysis of randomised controlled trials. BMJ Open 2016;6: e010524. doi:10.1136/ bmjopen-2015-010524

- Prepublication history and additional material is available. To view please visit the journal (http://dx.doi.org/ 10.1136/bmjopen-2015010524).

Received 17 November 2015 Revised 18 March 2016 Accepted 15 April 2016

CrossMark

\footnotetext{
${ }^{1}$ State Key Laboratory of Quality Research in Chinese Medicine, Institute of Chinese Medical Sciences, University of Macau, Macao, China ${ }^{2}$ School of Informatics, University of Edinburgh, Edinburgh, UK
}

Correspondence to Dr Siu-wai Leung; swleung@umac.mo

\section{ABSTRACT}

Objective: The present study aimed to verify the effectiveness of self-monitoring of blood glucose (SMBG) in patients with non-insulin-treated type 2 diabetes (T2D).

Methods: A comprehensive literature search was conducted in PubMed, Cochrane Library, Web of Science, ScienceDirect and ClinicalTrials.gov from their respective inception dates to 26 October 2015. Eligible randomised controlled trials (RCTs) were included according to prespecified criteria. The quality of the included RCTs was evaluated according to the Cochrane Risk of Bias Tool, and the evidence quality of meta-analyses was assessed by the Grading of Recommendation, Assessment, Development, and Evaluation (GRADE) criteria. A meta-analysis of primary and secondary outcome measures was performed. Sensitivity and subgroup analyses were carried out to evaluate the robustness and heterogeneity of the findings. Begg's and Egger's tests were used to quantify publication biases.

Results: A total of 15 RCTs, comprising 3383 patients with non-insulin-treated T2D, met the inclusion criteria. The SMBG intervention improved glycated haemoglobin $(\mathrm{HbA} 1 \mathrm{c})$ (mean difference -0.33; 95\% $\mathrm{Cl}-0.45$ to $-0.22 ; p=3.0730 \mathrm{e}-8 ; n=18)$, body mass index (BMI; $-0.65 ;-1.18$ to $-0.12 ; p=0.0164 ; n=9$ ) and total cholesterol (TC; $-0.12 ;-0.20$ to -0.04 ; $\mathrm{p}=0.0034 ; n=8$ ) more effectively than the control in overall effect. The sensitivity analysis revealed little difference in overall effect, indicating the robustness of the results. SMBG moderated $\mathrm{HbA1c}$ levels better than the control in all subgroup analyses. Most of the RCTs had high risk of bias in blinding, while the overall quality of evidence for $\mathrm{HbA1c}$ was moderate according to the GRADE criteria. Publication bias was moderate for BMI.

Conclusions: SMBG improved $\mathrm{HbA1c}$ levels in the short term ( $\leq 6$-month follow-up) and long term ( $\geq 12$-month follow-up) in patients with T2D who were not using insulin.

Trial registration number: CRD42015019099.

\section{Strengths and limitations of this study}

This meta-analysis provides the latest evidence for the effect of self-monitoring of blood glucose (SMBG) on non-insulin-treated type 2 diabetes (T2D) to resolve the controversy among previous studies, including the latest Cochrane review. This study is clinically relevant and significant because of the following reasons:

- It is the most comprehensive meta-analysis to include the latest randomised controlled trials (RCTs) and evaluates more clinical indexes (including systolic blood pressure (SBP), diastolic blood pressure (DBP), weight, body mass index (BMI), triglyceride (TG), total cholesterol (TC) and waist circumference) than previous meta-analyses.

- It is the first meta-analysis to investigate the association of geographical regions (Asia and America-Europe) with the effectiveness of SMBG.

- It provides a clear demonstration that SMBG is significantly effective in controlling glycated haemoglobin $(\mathrm{HbA1C}), \mathrm{BMI}$ and TC levels in the short term, while a clinically significant change in the long term is only observed in patients with newly diagnosed T2D with mildly elevated $\mathrm{HbA1c}$.

\section{INTRODUCTION}

In 2011, £158 million was spent on selfmonitoring of blood glucose (SMBG) by the UK's National Health Service (NHS), accounting for $21 \%$ of diabetic prescription costs. ${ }^{1}$ The Centers for Disease Control and Prevention (CDC) estimated that the daily usage rate of SMBG in the USA was $63.4 \%$ among patients with diabetes, ${ }^{2}$ while the Diabetes Glycaemic Education and Monitoring (DiGEM) trial reported that the 12-month costs of SMBG were similar in both less intensive (£92) and more intensive (£84) SMBG groups. ${ }^{3}$ SMBG has been reported 
effective in the management of type 1 diabetes mellitus. ${ }^{4}$ However, there remains a lack of consensus over the effectiveness of SMBG for self-management of patients with non-insulin-treated type 2 diabetes (T2D), despite several meta-analyses having investigated the issue. ${ }^{6-13}$ Given the high costs incurred during SMBG, if the technology was found to be ineffective in the treatment of patients with T2D without insulin administration, the expenditure would be in vain. Conversely, if SMBG was proven effective, over 200 million patients with T2D living in low-income and middle-income countries could be advised to manage their disease through SMBG as a relatively low-cost health technology. Several meta-analyses $^{7} 12 \quad 13$ deemed that SMBG was effective in decreasing glycated haemoglobin (HbAlc) levels in patients with T2D. Others ${ }^{6} 8$ found that the SMBG group did not fare significantly better than the control group, but varied in the length of follow-up. The latest meta-analysis was a Cochrane review published in 2012, which suggested that SMBG had a minimal effect on improving glucose control at 6 months (mean difference -0.26 ; $95 \%$ CI -0.39 to -0.13 ), but the effect disappeared after 12 months $(-0.13 ;-0.31$ to 0.04$)$; thus, the clinical benefit was limited. ${ }^{6}$ Since then, more randomised controlled trials (RCTs) ${ }^{14-17}$ have been published, of which three ${ }^{14} 1517$ suggested that SMBG improved diabetic control beyond the levels estimated by the last Cochrane review $^{6}$ (ie, at 6 and 12 months), and one ${ }^{16}$ reported no significant differences between SMBG and usual care for changes in HbAlc levels. Regardless of the controversial results of these previous reviews and recent RCTs, the last Cochrane review is already outdated. Therefore, the present study aimed to re-estimate the effects of SMBG in patients with non-insulin-treated T2D through a meta-analysis of RCTs. Given the significant efficacy and safety differences observed among ethnic groups in the DURAbility of Basal versus Lispro mix $75 / 25$ insulin Efficacy (DURABLE) trial, ${ }^{18}$ the present study also investigated the possible associations of ethnicity and living environment with SMBG.

\section{METHODS}

The present systematic review was conducted in accordance with the protocol registered in the International prospective register of systematic reviews (PROSPERO) (registration number: CRD42015019099) and the Preferred Reporting Items for Systematic Reviews and Meta-analyses (PRISMA) guidelines. ${ }^{19}$

\section{Eligibility criteria}

Included studies met the following criteria: (1) RCTs comparing SMBG with usual care (or no SMBG) in patients with non-insulin-treated T2D above 18 years of age; (2) RCTs reporting HbAlc levels; and (3) RCTs reporting fasting plasma glucose (FPG), body mass index (BMI; calculated as weight in kilograms divided by square of the height in metres), diastolic blood pressure
(DBP), systolic blood pressure (SBP), high-density lipoprotein (HDL), low-density lipoprotein (LDL), triglycerides (TGs), total cholesterol (TC), waist circumference (WC) and weight (if available). The primary outcome measure in this study was HbAlc improvement and the secondary outcome measures were FPG, BMI, DBP, SBP, HDL, LDL, TG, TC, WC and weight improvement. Meeting summaries and abstracts were excluded.

\section{Literature search and study selection}

Two reviewers (HZ and YZ) searched four bibliographical databases, namely PubMed, Web of Science, ScienceDirect and Cochrane Library, from their respective inception dates to 26 October 2015. Google Scholar and ClinicalTrials.gov were adopted as additional database sources. Manual searching was used to retrieve additional records from relevant studies. The search strategies were specified according to the database used. Basic search terms included 'self-monitoring of blood glucose', 'type 2 diabetes' and 'non-insulin' in the title, abstract and/or keywords. The specific search strategies used were:

PubMed: ((self-monitoring of blood glucose[Title/ Abstract] OR SMBG[Title/Abstract]) AND (type 2 diabetes [Title/Abstract] OR T2D [Title/Abstract])) AND ( (no insulin [Title/Abstract] OR insulin [Title/ Abstract]) OR non-insulin [Title/Abstract]).

Web of Science: $\mathrm{TS}=(\mathrm{SMBG}$ OR self-monitoring of blood glucose) AND TS=(T2D OR type 2 diabetes) AND $\mathrm{TS}=$ (non-insulin OR using insulin OR no insulin).

ScienceDirect: TITLE-ABSTR-KEY ((SMBG OR selfmonitoring of blood glucose) AND (T2D OR type 2 diabetes) AND (non-insulin OR using insulin OR no insulin)).

The Cochrane Library: self-monitoring of blood glucose or SMBG in Title, Abstract, Keywords AND no insulin OR non-insulin in Title, Abstract, Keywords AND type 2 diabetes OR T2D in Title, Abstract, Keywords.

Two reviewers ( $\mathrm{HZ}$ and $\mathrm{YZ}$ ) independently performed the literature searches and selection. The articles retrieved by one reviewer were crosschecked by the other. Disagreements were resolved by group discussion or in consultation with a third reviewer (S-wL).

\section{Data extraction and quality assessment}

Two reviewers (HZ and YZ) independently extracted data on study characteristics (such as author, publication year, sample size and intervention) and outcome measures from the eligible studies. The extracted data were crosschecked and any disagreement was resolved by discussion between the two reviewers ( $\mathrm{HZ}$ and $\mathrm{YZ})$; a third reviewer $(\mathrm{S}-\mathrm{wL})$ was consulted when necessary. The quality of the included RCTs was evaluated according to the Cochrane Risk of Bias Tool, ${ }^{20}$ and the evidence quality of meta-analyses was assessed using the Grading of Recommendation, Assessment, Development, and Evaluation (GRADE) criteria. $^{21}$ 


\section{Meta-analysis}

Extracted data were transferred to the 'metafor' package ${ }^{22}$ in $\mathrm{R}$ software for meta-analysis with a random-effects model. Continuous outcome measures were calculated and represented as mean differences (MDs) and their 95\% CIs. Heterogeneity among RCTs was evaluated with $\mathrm{I}^{2}$ statistics. $\mathrm{p}$ Values $<0.05$ were considered statistically significant.

\section{Sensitivity and subgroup analyses}

A sensitivity analysis was performed on sample size to assess the robustness of the findings. Sample size is a dominant factor that affects precision in determining overall effects. Thus, the meta-analysis was repeated after excluding studies with sample sizes of 99 or less. Subgroup analyses were performed to explain the heterogeneity in terms of geographical region, follow-up period and diabetes history, according to the characteristics of the included studies. The Mann-Whitney-Wilcoxon test was used to determine the differences across subgroups.

\section{Adverse events analysis}

Meta-analyses on the overall adverse effects in terms of ORs and their 95\% CIs were performed with a random-effects model.

\section{Publication bias and metaregression}

Funnel plots were generated to visualise any possible publication bias. Begg's rank correlation test ${ }^{23}$ and Egger's regression test ${ }^{24}$ were performed to evaluate the statistical significance of the publication bias. A metaregression with a mixed-effects model employing the restricted maximum likelihood (REML) estimation was performed to examine the possible relationships between the overall effects and factors such as sample size, history of diabetes, follow-up period and publication year.

\section{Classification tree}

A classification tree was manually constructed to indicate who might benefit from SMBG. The classification tree example in online supplementary figure S3 can be interpreted as, 'the mean starting HbAlc of tested patients was no less than $8 \%$, then if the follow-up period of trials was short (no more than 6 months), then if the tested patients from Asia, then if the patients is newly diagnosed'. Unpaired t-tests were performed with $\mathrm{R}$ software for two-group comparisons.

\section{RESULTS}

\section{Study characteristics}

A total of 1533 records were retrieved in accordance with the specified search strategies from PubMed $(n=246)$, Web of Science $(n=400)$, ScienceDirect $(n=352)$, Cochrane Library $(n=234)$, Google Scholar $(n=266)$ and ClinicalTrials.gov $(n=35)$. Following removal of duplicates, 683 records were screened, of which 50 were assessed for eligibility. Finally, 15 records were included after full-text assessment (see online supplementary table S1). Figure 1 shows the study selection process and the reasons for exclusion. The 15 studies ${ }^{14-17} 25-35$ meeting the inclusion criteria comprised a total of 3383 patients with non-insulin-treated T2D. Among the 15 studies, 13 studies had HbAlc as their primary outcome and the other two ${ }^{16}{ }^{17}$ had HbA1c as a secondary outcome. A total of $9^{15} \quad 25 \quad 26 \quad 29-31 \quad 33-35$ of the 15 studies were two-armed trials comparing SMBG with usual care or no SMBG, while 6 studies $^{14} 16 \quad 17272832$ were three-armed trials. Among the three-armed trials, three ${ }^{1628} 32 \mathrm{com}-$ pared SMBG and self-monitoring of urine glucose (SMUG) with usual care, two ${ }^{1727}$ compared less intensive SMBG and more intensive SMBG with a control group, and one ${ }^{14}$ compared fingertip SMBG and palm SMBG with a control group.

\section{Overall effects}

A meta-analysis was performed on the primary outcome measure of $\mathrm{HbAlc}$ and secondary outcome measures of FPG, BMI, DBP, SBP, HDL, LDL, TG, TC, WC and body weight (table 1). The SMBG group was shown to perform better for HbAlc (MD $-0.33 ; 95 \%$ CI -0.45 to $-0.22 ; \mathrm{p}=3.0730 \mathrm{e}-8 ; \mathrm{n}=18)$, BMI $(-0.65 ;-1.18$ to -0.12 ; $\mathrm{p}=0.0164 ; \mathrm{n}=9)$, TC $(-0.12 ;-0.20$ to $-0.04 ; \mathrm{p}=0.0034$; $\mathrm{n}=8)$ and WC $(-2.22 ;-4.40$ to $-0.03 ; \mathrm{p}=0.0474 ; \mathrm{n}=5)$ compared with the control group. There were no significant differences with regard to FPG, DBP, SBP, HDL, LDL, TG and weight between the SMBG and control groups. Among the studies, significant heterogeneity was found for HbAlc $\left(I^{2}=63 \%, p=0.0002\right)$, BMI $\quad\left(I^{2}=89 \%\right.$, $\mathrm{p}=0.0009)$, LDL $\left(\mathrm{I}^{2}=89 \%, \mathrm{p}=0.0009\right)$ and SBP $\left(\mathrm{I}^{2}=65 \%\right.$, $\mathrm{p}=0.0100$ ). A forest plot of HbAlc is shown in figure 2 to allow visual assessment of the heterogeneity.

\section{Quality of RCTs and meta-analyses}

Online supplementary figures S1 and S2 show the quality of the included RCTs assessed by the Cochrane Risk of Bias Tool. Almost 50\% of the RCTs did not provide adequate information about random sequence generation, allocation concealment and selective reporting. All of the included studies had high risk of bias in blinding of participants, because the participants could not be blinded to the intervention, and only four RCTs had low risk of bias in blinding of outcome assessment. Other key aspects identified among the RCTs were mostly low for risk of bias. Table 1 shows the evidence quality of the meta-analyses evaluated by the GRADE criteria. The overall quality of evidence for HbAlc was moderate, with significant heterogeneity across studies $\left(\mathrm{I}^{2}=63 \% ; \mathrm{p}=0.0002 ; \mathrm{n}=18\right)$. The quality of evidence for FPG, TG and TC was rated as high.

\section{Sensitivity analysis}

A sensitivity analysis was performed on studies with sample sizes of 100 or above to test the robustness of the overall effects. As shown in table 2, there were minor 


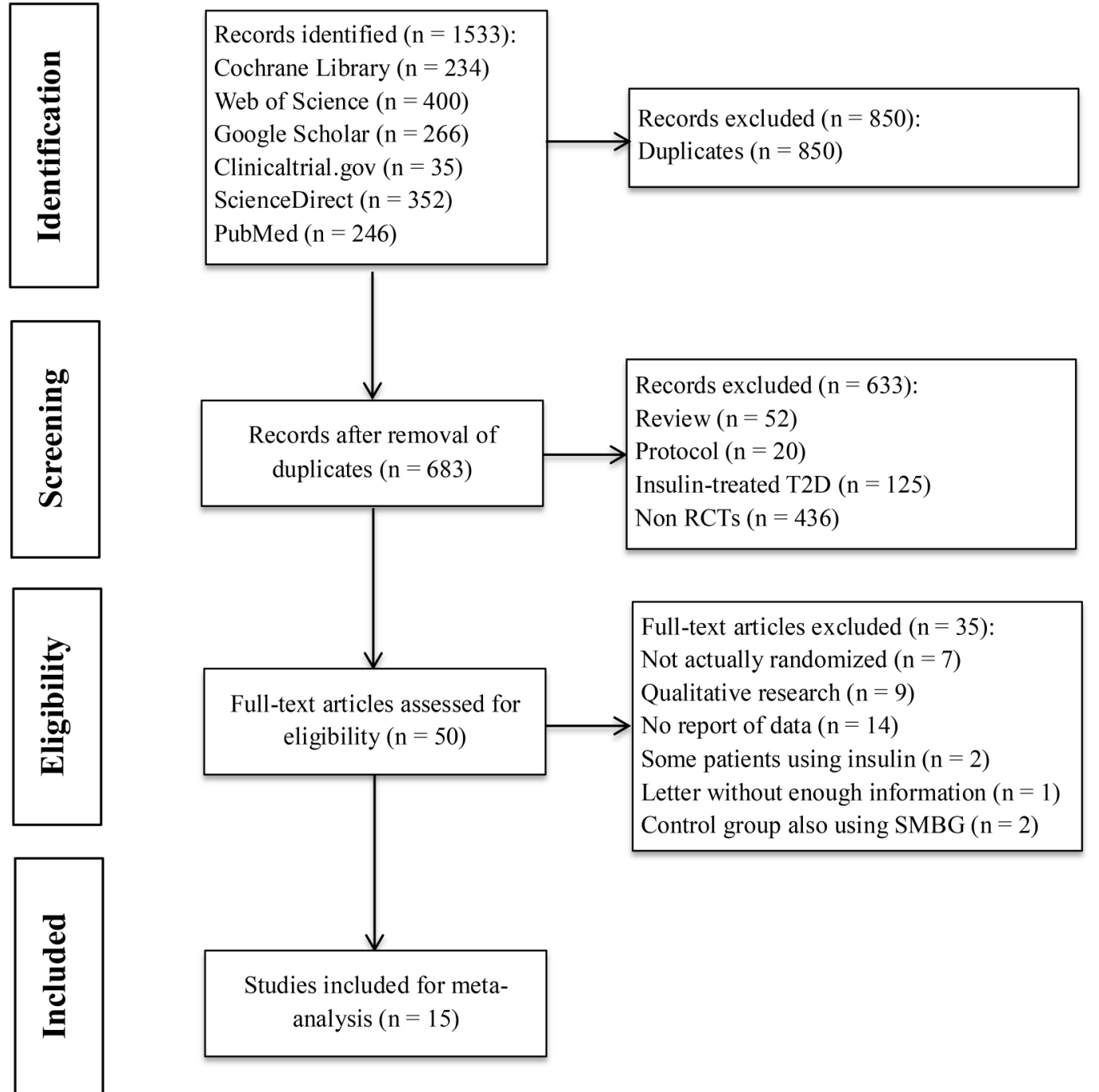

Figure 1 Flow chart of study selection. The process of study selection included literature search, abstract screening, eligibility evaluation and study inclusion for meta-analysis. RCT, randomised controlled trial; SMBG, self-monitoring of blood glucose; T2D, type 2 diabetes.

changes in the significance of SMBG for HbA1c, BMI, $\mathrm{TC}$ and $\mathrm{WC}$, indicating the robustness of the overall effects. A forest plot of HbAlc is shown in figure 3.

\section{Subgroup analyses}

Subgroup analyses stratified trials according to geographical regions to examine the source of heterogeneity among the RCTs. The studies were divided into two groups: one comprising study populations from Asian countries (Asia group) and the other comprising study populations from America and Europe (AmericaEurope group) (see online supplementary table S2). SMBG significantly improved HbA1c levels and BMI regardless of the groups. Subgroup analyses were also performed on studies with follow-up periods of 12 months or more to test the long-term effects of SMBG (see online supplementary table S3), and studies with follow-up periods of 6 months or less to test the short-term effects. SMBG moderated HbAlc levels better than the control group in the short term and long term, while both interventions were equivalent in performance for all aspects except for BMI and TC. Subgroup analyses based on history of diabetes ( $\geq 12$ months or newly diagnosed) were also performed (see online supplementary table S4). The SMBG group outperformed the control group for $\mathrm{HbAlc}$, BMI and TC, indicating that SMBG was effective in controlling blood glucose in the later phase of diabetes. Only two trials with three substudies were conducted in newly diagnosed patients, and these were analysed in the Cochrane review. ${ }^{6}$

\section{Adverse events}

A total of 7 of the 15 studies reported adverse events. As shown in online supplementary table S5, the most commonly reported adverse event was hypoglycaemia. The incidence of hypoglycaemia was higher in the SMBG group than in the control group, but the rate of hypoglycaemic episodes per patient was higher in the control 
Table 1 Results of overall efficacy

\begin{tabular}{|c|c|c|c|c|c|c|c|}
\hline \multirow[b]{2}{*}{ Outcome } & \multirow[b]{2}{*}{ Substudies (n) } & \multirow{2}{*}{$\begin{array}{l}\text { Pooled } \\
\text { sample size }\end{array}$} & \multicolumn{2}{|c|}{ Heterogeneity } & \multicolumn{2}{|l|}{ Overall effect } & \multirow{2}{*}{$\begin{array}{l}\text { Quality of evidence } \\
\text { assessed by } \\
\text { GRADE* }^{\text {GRA }}\end{array}$} \\
\hline & & & $I^{2}(\%)$ & p Value & MD (95\% Cl) & p Value & \\
\hline $\mathrm{HbA1c}$ & 18 & 3383 & 63 & 0.0002 & $-0.33(-0.45$ to -0.22$)$ & $3.0730 \mathrm{e}-8$ & $\oplus \oplus \oplus \ominus$ Moderate \\
\hline FPG & 3 & 1366 & 0 & 0.6739 & $-0.23(-0.56$ to 0.11$)$ & 0.1804 & $\oplus \oplus \oplus \oplus$ High \\
\hline BMI & 9 & 1391 & 89 & 0.0009 & $-0.65(-1.18$ to -0.12$)$ & 0.0164 & $\oplus \oplus \ominus \ominus$ Low \\
\hline DBP & 7 & 1740 & 59 & 0.0419 & $-0.86(-2.21$ to 0.48$)$ & 0.2083 & $\oplus \oplus \ominus \ominus$ Low \\
\hline SBP & 7 & 1740 & 65 & 0.0100 & $-1.08(-3.46$ to 1.29$)$ & 0.3710 & $\oplus \oplus \oplus \ominus$ Moderate \\
\hline HDL & 4 & 446 & 67 & 0.0175 & $0.04(-0.07$ to 0.14$)$ & 0.5257 & $\oplus \oplus \oplus \ominus$ Moderate \\
\hline LDL & 3 & 384 & 89 & 0.0009 & $-0.13(-0.66$ to 0.39$)$ & 0.6140 & $\oplus \oplus \oplus \ominus$ Moderate \\
\hline TG & 6 & 736 & 0 & 0.8983 & $0.02(-0.15$ to 0.20$)$ & 0.7990 & $\oplus \oplus \oplus \oplus$ High \\
\hline $\mathrm{TC}$ & 8 & 1342 & 0 & 0.5220 & $-0.12(-0.20$ to -0.04$)$ & 0.0034 & $\oplus \oplus \oplus \oplus$ High \\
\hline Weight & 8 & 1814 & 0 & 0.9838 & $-0.29(-0.62$ to 0.05$)$ & 0.0958 & $\oplus \oplus \oplus \ominus$ Moderate \\
\hline WC & 5 & 513 & 0 & 0.4779 & $-2.22(-4.40$ to -0.03$)$ & 0.0474 & $\oplus \oplus \oplus \ominus$ Moderate \\
\hline
\end{tabular}

*Grading of Recommendation, Assessment, Development, and Evaluation (GRADE). ${ }^{21}$

BMI, body mass index; DBP, diastolic blood pressure; FPG, fasting plasma glucose; HbA1c, glycated haemoglobin; HDL, high-density lipoprotein; LDL, low-density lipoprotein; MD, mean difference; SBP, systolic blood pressure; TC, total cholesterol; TG, triglyceride; WC, waist circumference.

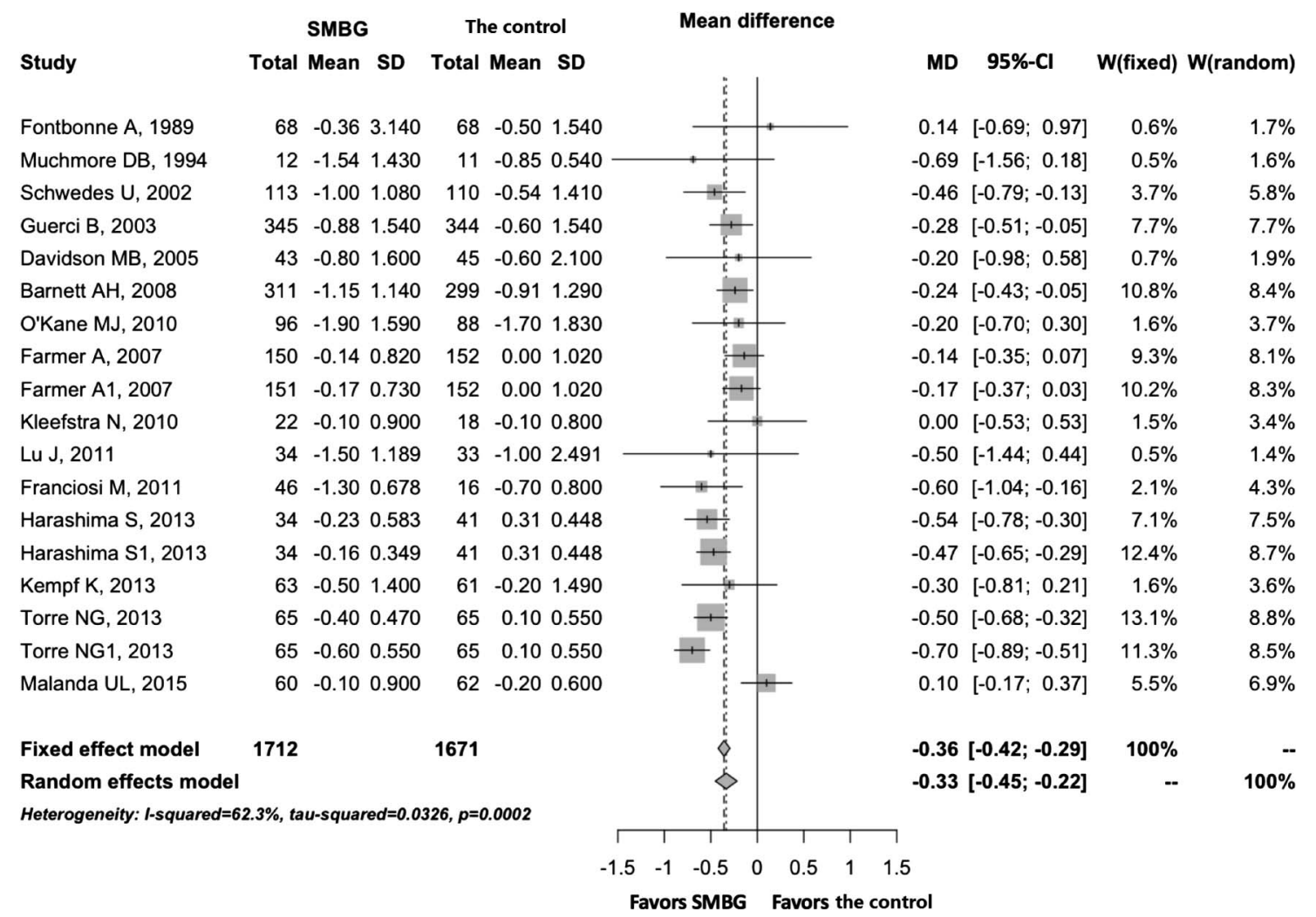

Figure 2 Forest plot of $\mathrm{HbA1c}$ for overall effect, showing the mean differences between SMBG and the control with corresponding $95 \%$ Cls in the individual studies. HbA1c, glycated haemoglobin; SMBG, self-monitoring of blood glucose.

group. A statistical analysis of adverse events was not performed because the data available from the RCT reports were insufficient in terms of quantity.

\section{Publication bias}

Funnel plots of the outcomes for HbA1c, FPG, BMI, DBP, SBP, HDL and LDL showed various levels of asymmetries across the studies, indicating possible publication bias. Begg's rank correlation test and Egger's regression test detected moderate publication bias for BMI (see online supplementary table S6). The results of the funnel plots, Egger's test and Begg's test are not presented in the main text, because they are not recommended for fewer than 10 studies based on a high risk of inaccurate interpretation. 
Table 2 Sensitivity analysis on studies with sample sizes of 100 or above

\begin{tabular}{|c|c|c|c|c|c|c|}
\hline \multirow[b]{2}{*}{ Outcome } & \multirow[b]{2}{*}{ Substudies (n) } & \multirow[b]{2}{*}{ Pooled sample size } & \multicolumn{2}{|c|}{ Heterogeneity } & \multicolumn{2}{|l|}{ Overall effect } \\
\hline & & & $I^{2}(\%)$ & p Value & MD (95\% Cl) & p Value \\
\hline $\mathrm{HbA1c}$ & 11 & 2953 & 72 & $<0.0001$ & $-0.29(-0.44$ to -0.14$)$ & 0.0001 \\
\hline FPG & 2 & 1299 & 0 & 0.4186 & $-0.21(-0.56$ to 0.14$)$ & 0.2384 \\
\hline BMI & 6 & 1174 & 97 & 0.0025 & $-0.89(-1.88$ to 0.09$)$ & 0.0757 \\
\hline DBP & 6 & 1678 & 65 & 0.0269 & $-0.82(-2.24$ to 0.61$)$ & 0.2628 \\
\hline SBP & 7 & 1740 & 65 & 0.0100 & $-1.08(-3.46$ to 1.29$)$ & 0.3710 \\
\hline HDL & 3 & 384 & 79 & 0.0066 & $0.04(-0.11$ to 0.18$)$ & 0.6268 \\
\hline LDL & 3 & 384 & 89 & 0.0009 & $-0.13(-0.66$ to 0.39$)$ & 0.6140 \\
\hline TG & 4 & 607 & 0 & 0.6740 & $0.03(-0.15$ to 0.21$)$ & 0.7529 \\
\hline TC & 6 & 1213 & 7 & 0.3190 & $-0.11(-0.21$ to -0.01$)$ & 0.0254 \\
\hline Weight & 5 & 1641 & 0 & 0.9447 & $-0.28(-0.62$ to 0.07$)$ & 0.1172 \\
\hline WC & 3 & 384 & 13 & 0.2934 & $-3.15(-6.46$ to 0.17$)$ & 0.0630 \\
\hline
\end{tabular}

BMI, body mass index; DBP, diastolic blood pressure; FPG, fasting plasma glucose; HbA1c, glycated haemoglobin; HDL, high-density lipoprotein; LDL, low-density lipoprotein; MD, mean difference; SBP, systolic blood pressure; TC, total cholesterol; TG, triglyceride; WC, waist circumference.

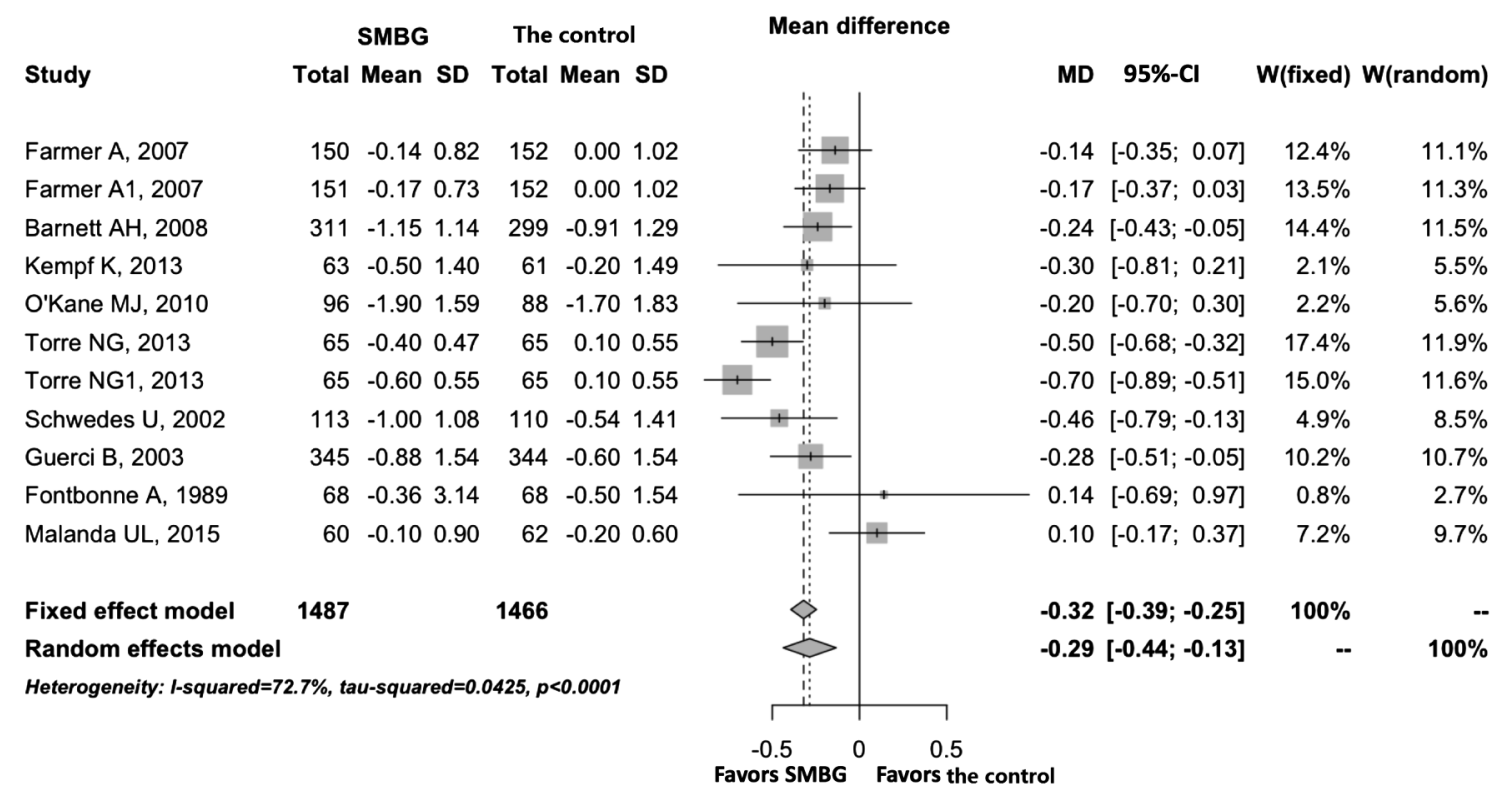

Figure 3 Forest plot of $\mathrm{HbA} 1 \mathrm{c}$ for sensitivity analysis, showing the mean differences between SMBG and the control with corresponding $95 \% \mathrm{Cls}$ in the individual studies whose sample sizes were bigger than 99. HbA1c, glycated haemoglobin; SMBG, self-monitoring of blood glucose.

\section{Metaregression}

SMBG performed better for HbAlc than the control under all tested conditions, and better for BMI and TC under most of the tested conditions but with heterogeneity. Therefore, a metaregression was performed to analyse the relationships between these three outcomes and the study characteristics (including sample size, publication year, history of diabetes and follow-up) and test the source of the heterogeneity. As shown in table 3, there seemed to have significant relationships between BMI and history of diabetes, and between BMI and follow-up period. There was also a statistically significant association between history of diabetes and HbA1c.

\section{DISCUSSION}

The present meta-analysis included 15 RCTs, comprising 3383 participants, to assess the effectiveness of SMBG in patients with non-insulin-treated T2D with the primary outcome of HbAlc levels. A sensitivity analysis on sample size and subgroup analyses on geographical region and SMBG history were also performed, together with assessment of the associations of ethnicity and living environment with SMBG, because significant efficacy and safety differences were previously observed among racial/ethnic groups in the DURABLE trial. ${ }^{18}$ Various clinical indices, including SBP, DBP, body weight, BMI, TG, TC and WC, were also analysed and reported for the first time in this study. A comparison of 
Table 3 Metaregression analysis of the relationship between outcomes and characteristics of RCTs

\begin{tabular}{|c|c|c|c|c|c|}
\hline Outcome & Substudies (n) & Factor & Coefficient & $\mathbf{z}$ & p Value \\
\hline \multirow[t]{4}{*}{$\mathrm{HbA1c}$} & 18 & Sample size & 0.0004 & 1.0535 & 0.2921 \\
\hline & & History of diabetes & 0.2559 & 2.0006 & 0.0454 \\
\hline & & Follow-up & -0.0081 & -1.5508 & 0.1210 \\
\hline & & Publication year & -0.0079 & -0.6481 & 0.5169 \\
\hline \multirow[t]{4}{*}{ BMI } & 9 & Sample size & 0.0034 & 1.1169 & 0.2640 \\
\hline & & History of diabetes & 1.9653 & 3.4234 & 0.0006 \\
\hline & & Follow-up & -0.0705 & -2.3762 & 0.0175 \\
\hline & & Publication year & -0.1805 & -1.4780 & 0.1394 \\
\hline \multirow[t]{4}{*}{ TC } & 8 & Sample size & -0.0001 & -0.3244 & 0.7456 \\
\hline & & History of Diabetes & -0.0249 & -0.2167 & 0.8284 \\
\hline & & Follow-up & 0.0011 & 0.2634 & 0.7923 \\
\hline & & Publication year & -0.0021 & -1.2347 & 0.2169 \\
\hline
\end{tabular}

BMI, body mass index; HbA1c, glycated haemoglobin; TC, total cholesterol.

the RCTs included in this meta-analysis with those included in some previous meta-analyses is presented (see online supplementary table S7).

The results for overall effects suggested that use of SMBG by patients with non-insulin-treated T2D led to a significant reduction in HbAlc levels (MD -0.33; $95 \%$ CI -0.45 to $-0.22 ; \mathrm{p}=3.0730 \mathrm{e}-8 ; \mathrm{n}=18$ ), which is consistent with previous systematic reviews. ${ }^{712} 13$ The sensitivity analysis of studies with large sample sizes showed that SMBG could help reduce HbAlc levels in a robust manner. The subgroup analysis of geographical regions revealed that significant decreases in HbAlc levels were observed in the Asia and America-Europe groups. SMBG improved the HbA1c levels in the short term $(\leq 6$ months), but also had long-term effects ( $\geq 12$-month follow-up), being different from the Cochrane review. ${ }^{6}$ Significant reductions in $\mathrm{HbAlc}$ levels were also found in newly diagnosed patients and patients with diabetes for more than 12 months, indicating that SMBG seemed to benefit patients with T2D regardless of diabetes duration. The quality of evidence for HbAlc was rated as moderate, which was the same as the last Cochrane review. ${ }^{6}$ The factors influencing the evidence quality mainly appeared to be small effect size and significant heterogeneity in this meta-analysis.

In addition to HbA1c, significant reductions in several clinical indices, including BMI, TC and WC, were found in various analyses, indicating that SMBG does not simply function as a monitoring tool, but forms a component of a complex intervention capable of improving overall glycaemic control. Despite BMI and TC being the only clinical indices to show significant reductions following SMBG in patients diagnosed with T2D for over 12 months, the present results suggest that SMBG may have long-term effects on the control of BMI and TC in patients with T2D. Reductions in BMI and TC were also observed in other subgroup and sensitivity analyses. The reductions ranged from -0.87 to -0.24 for $\mathrm{BMI}$ and from -0.12 to $-0.11 \mathrm{mmol} / \mathrm{L}$ for $\mathrm{TC}$, indicating that SMBG might be useful for obese patients. Although SMBG was helpful for BMI, the evidence quality for BMI was low, and moderate publication bias was also observed. Thus, more RCTs are warranted for the effect of SMBG on BMI. Any observed reductions in the remaining indices had no clinical significance, in agreement with the findings of Farmer et al. ${ }^{27}$

The above-discussed evidence indicates that SMBG helped to improve glycaemic control, but under a single specific test condition. Thus, combinations of two or more test conditions are required to address the issue of what type of patient under what kind of intervention would achieve improvement of glycaemic control by SMBG. A classification tree method was adopted to show the influence of baseline HbAlc, follow-up period, geographical region and diabetes duration on SMBG. As shown in online supplementary figure S3, SMBG improved glycaemic control regardless of baseline HbA1c, while SMBG was significantly more effective in control patients with lower HbAlc $(<8 \%)$ than in those with higher HbAlc $(\geq 8 \%)$. Among the patients with lower HbA1c, SMBG improved glycaemic control in the short term and long term, and performed better in the former. Furthermore, SMBG might have long-term effects in the improvement of glycaemic control in newly diagnosed patients with T2D with lower HbA1c. All of the evidence shown in online supplementary figure S3 indicates that SMBG has a short-term effect in T2D, and that a clinically significant change in the long term is only observed in newly diagnosed patients with T2D with lower HbAlc.

Compared with the last Cochrane review, ${ }^{6}$ there are several differences. First, RCTs involving comparisons between SMBG and SMUG (as a control) or between SMUG and usual care (SMUG as an intervention) were not included in the present study, because SMBG was preferred for perceived accuracy and usefulness, ${ }^{10}$ and SMUG was associated with renal glucose threshold, combined diseases and drugs, which would not allow us to distinguish among hypoglycaemia, normoglycaemia and mild hyperglycaemia. Second, in our analysis, SMBG was significantly better than usual care in the short term and long term, while SMBG was better in the short term in the last Cochrane review. ${ }^{6}$ 
There are several limitations to this meta-analysis. First, the sample sizes in some of the included studies were too small to confidently exclude a difference in response in the reduction of HbAlc and clinical indices between the SMBG and control groups, and this might be a source of heterogeneity. Second, the heterogeneities of the pooled effect sizes on HbAlc were high, and according to previous reviews ${ }^{8-10}$ and the RCTs included in this meta-analysis, the most important heterogeneities might arise through the intensity of education, intervention components coupled to SMBG and frequency of SMBG testing. Third, there were no properly collected data for a statistical analysis of adverse events and economic assessment. Fourth, potential synonyms for SMBG and diabetes were not included in the strategy, and EMBASE was not searched, which may have led to relevant papers being missed and possible publication bias. Finally, although SMBG did not achieve the $0.5 \%$ reduction in HbAlc deemed a clinically significant change, ${ }^{10}$ it significantly reduced the HbAlc level under all tested conditions. Last, the present evidence warrants further research, because some outcome measures were reported by fewer studies and the potential associations among the outcome measures remain unclear.

\section{CONCLUSION}

In patients with T2D who were not using insulin, SMBG improved the HbA1c level in the short term and long term.

Acknowledgements This work originated from a postgraduate course project supervised by the corresponding author. After completing the course, the leader of this project group (YZ) and the other two authors of this manuscript found errors in study design and data; thus, they redesigned the study and criteria for study eligibility. The authors are grateful to the other project team members Runmiao Wang and Zichao Gao for their help in the original group project, although they cannot be included as authors according to the ICMJE's authorship guideline.

Contributors S-wL is the guarantor of this work and conceived the metaanalysis. S-wL, $\mathrm{HZ}$ and $\mathrm{YZ}$ designed the protocol. $\mathrm{HZ}$ and $\mathrm{YZ}$ searched the databases, assessed and selected studies, and extracted and analysed the data from the selected studies according to the eligibility criteria. $\mathrm{HZ}$ and $\mathrm{YZ}$ interpreted the data and drafted a report on the findings. S-wL revised the manuscript for submission. All authors read and approved the final version of the manuscript.

Funding This work was supported by the University of Macau (MYRG2014-00117-ICMS-QRCM).

Competing interests None declared.

Provenance and peer review Not commissioned; externally peer reviewed.

Data sharing statement No additional data are available.

Open Access This is an Open Access article distributed in accordance with the Creative Commons Attribution Non Commercial (CC BY-NC 4.0) license, which permits others to distribute, remix, adapt, build upon this work noncommercially, and license their derivative works on different terms, provided the original work is properly cited and the use is non-commercial. See: http:// creativecommons.org/licenses/by-nc/4.0/

\section{REFERENCES}

1. National Prescribing Centre. Data focused commentary: type 2 diabetes: selfmonitoring blood glucose. 2012. http://www.npc.nhs.uk/ therapeutics/cardio/ diabetes_2/resources/dfc_self_monitoring_of_ blood glucose.pdf (accessed 25 Feb 2015).

2. Centers for Disease Control and Prevention. Prevalence of self-reported cardiovascular disease among persons aged $>$ or $=35$ years with diabetes--United States, 1997-2005. MMWR Morb Mortal Wkly Rep 2007;56:1129-32.

3. Simon J, Gray A, Clarke P, et al. Cost effectiveness of self monitoring of blood glucose in patients with non-insulin treated type 2 diabetes: economic evaluation of data from the DiGEM trial. $B M J$ 2008;336:1177-80.

4. The effect of intensive treatment of diabetes on the development and progression of long-term complications in insulin-dependent diabetes mellitus. The Diabetes Control and Complications Trial Research Group. N Engl J Med 1993;329:977-86.

5. Bode BW, Gross TM, Thornton KR, et al. Continuous glucose monitoring used to adjust diabetes therapy improves glycosylated hemoglobin: a pilot study. Diabetes Res Clin Pract 1999;46:183-90.

6. Malanda U, Welschen L, Riphagen I, et al. Self-monitoring of blood glucose in patients with type 2 diabetes mellitus who are not using insulin. Cochrane Database Syst Rev 2012;1:CD005060.

7. Sarol JN, Nicodemus NA, Tan KM, et al. Self-monitoring of blood glucose as part of a multi-component therapy among non-insulin requiring type 2 diabetes patients: a meta-analysis (1966-2004). Curr Med Res Opin 2005;21:173-84.

8. Greater Los Angeles Veterans Affairs Healthcare System/Southern California/RAND evidence-based Practice Center. Self-monitoring of blood glucose in patients with type 2 diabetes mellitus: meta analysis of effectiveness. 2007; Sep:1-46.

9. Mcintosh B, Yu C, Lal A, et al. Efficacy of self-monitoring of blood glucose in patients with type 2 diabetes mellitus managed without insulin: a systematic review and meta-analysis. Open Med 2010;4: e102-13.

10. Clar C, Barnard K, Cummins E, et al. Self-monitoring of blood glucose in type 2 diabetes: systematic review. Health Technol Assess 2010;14:1-140.

11. Farmer AJ, Perera R, Ward A, et al. Meta-analysis of individual patient data in randomised trials of self monitoring of blood glucose in people with non-insulin treated type 2 diabetes. BMJ 2012;344:e486.

12. Poolsup N, Suksomboon N, Rattanasookchit S. Meta-analysis of the benefits of self-monitoring of blood glucose on glycemic control in type 2 diabetes patients: an update. Diabetes Technol Ther 2009:11:775-84.

13. John AS, Davis WA, Price CP, et al. The value of self-monitoring of blood glucose: a review of recent evidence. $J$ Diabetes Complications 2010;24:129-41.

14. Harashima S, Toru F, Mayumi S, et al. Self-monitoring of blood glucose (SMBG) improves glycaemic control in oral hypoglycaemic agent (OHA)-treated type 2 diabetes (SMBG-OHA study). Diabetes Metab Res Rev 2013;29:77-84.

15. Kempf K, Tankova T, Martin S. ROSSO-in-praxi-international: longterm effects of self-monitoring of blood glucose on glucometabolic control in patients with type 2 diabetes mellitus not treated with insulin. Diabetes Technol Ther 2013;15:89-96.

16. Malanda UL, Bot SDM, Kostense PJ, et al. Effects of self-monitoring of glucose on distress and self-efficacy in people with non-insulintreated Type 2 diabetes: a randomized controlled trial. Diabet Med 2016;33:537-46.

17. García de la Torre N, Durán A, Valle L, et al. Early management of type 2 diabetes based on a SMBG strategy: the way to diabetes regression--the St Carlos study. Acta Diabetol 2013:50:607-14.

18. Davidson JA, Wolffenbuttel BH, Arakaki RF, et al. Impact of race/ ethnicity on efficacy and safety of two starter insulin regimens in patients with type 2 diabetes: a posthoc analysis of the DURABLE trial. Ethn Dis 2013;23:393-400.

19. Zhu HM, Zhu YN, Leung S, et al. PROSPERO International prospective register of systematic reviews. The efficacy of self-monitoring of blood glucose in non-insulin treated type 2 diabetics - a systematic review and meta-analysis of randomized controlled trials. 2015:2-4. http://www.crd.york.ac.uk/PROSPERO/display record.asp?ID=CRD42015019099. (accessed online Aug 2016).

20. Higgins JPT, Altman DG, Gøtzsche PC, et al. The Cochrane Collaboration's tool for assessing risk of bias in randomised trials. BMJ 2011;343:d5928.

21. Canfield SE, Dahm P. Rating the quality of evidence and the strength of recommendations using GRADE. World J Urol 2011:29:311-7.

22. Viechtbauer W. Conducting meta-analyses in $\mathrm{R}$ with the metafor package. J Stat Softw 2010;36:1-48.

23. Begg CB, Mazumdar M. Operating characteristics of a rank correlation test for publication bias. Biometrics 1993;50:1088-101. 
24. Egger M, Smith GD, Schneider M, et al. Bias in meta-analysis detected by a simple, graphical test. BMJ 1997;315:629-34.

25. Barnett AH, Krentz AJ, Strojek K, et al. The efficacy of self-monitoring of blood glucose in the management of patients with type 2 diabetes treated with a gliclazide modified release-based regimen. A multicentre, randomized, parallel-group, 6-month evaluation (DINAMIC 1 study). Diabetes Obes Metab 2008;10:1239-47.

26. Davidson MB, Castellanos M, Kain D, et al. The effect of self monitoring of blood glucose concentrations on glycated hemoglobin levels in diabetic patients not taking insulin: a blinded, randomized trial. Am J Med 2005;118:422-5.

27. Farmer A, Wade A, Goyder E, et al. Impact of self monitoring of blood glucose in the management of patients with non-insulin treated diabetes: open parallel group randomised trial. BMJ 2007;335:132.

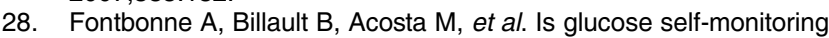
beneficial in noninsulin- treated diabetic patients? Results of a randomized comparative trial. Diabete Metab 1989;15:255-60.

29. Franciosi M, Lucisano G, Pellegrini F, et al. ROSES: role of self-monitoring of blood glucose and intensive education in patients with Type 2 diabetes not receiving insulin. A pilot randomized clinical trial. Diabet Med 2011;28:789-96.

30. Guerci B, Drouin P, Grange V, et al. Self-monitoring of blood glucose significantly improves metabolic control in patients with type 2 diabetes mellitus: the Auto-Surveillance Intervention Active (ASIA) study. Diabetes Metab J 2003;29:587-94.

31. Kleefstra N, Hortensius J, Logtenberg SJJ, et al. Self-monitoring of blood glucose in tablet-treated type 2 diabetic patients. Neth $\mathrm{J}$ Med 2010;68:311-16.

32. Lu J, Bu RF, Sun ZL, et al. Comparable efficacy of self-monitoring of quantitative urine glucose with self-monitoring of blood glucose on glycaemic control in non-insulin-treated type 2 diabetes. Diabetes Res Clin Pract 2011;93:179-86.

33. Muchmore DB, Springer J, Miller M. Self-monitoring of blood glucose in overweight type 2 diabetic patients. Acta Diabetol 1994;31:215-19.

34. O'Kane MJ, Bunting B, Copeland M, et al. Efficacy of self monitoring of blood glucose in patients with newly diagnosed type 2 diabetes (ESMON study): randomised controlled trial. BMJ 2008;336:1174-7.

35. Schwedes U, Siebolds M, Mertes G. Meal-related structured self-monitoring of blood glucose. Diabetes Care 2002;25:1928-32. 